\title{
Patient Centered Primary Care (PCPC): An Overview
}

Paul Grundy*

Global Director of Healthcare Transformation IBM, President PCPCC and Ambassador Healthcare, Denmark

*Corresponding author: Paul Grundy, Global Director of Healthcare Transformation IBM, Denmark, E-mail: pgrundy@us.ibm.com

Receiving date: April 27, 2015; Accepted date: September 18, 2015; Published date: September 21, 2015

Copyright: ( 2015 Grundy P. This is an open-access article distributed under the terms of the Creative Commons Attribution License, which permits unrestricted use, distribution, and reproduction in any medium, provided the original author and source are credited.

\begin{abstract}
There is no doubt that we face major problems both in health care quality and costs. The crucial question for the Employer (Buyer) is how to respond to these issues. There is a solution. Simply stated, the failure we are experiencing today is, basically, a failure in our primary care system. Therefore, any initiative that strives to improve the overall quality and cost of care must focus on primary care.
\end{abstract}

\section{Introduction}

The premise is that the problems of health care quality and cost are, to a very large extent, failures of the way we have organized and designed care in the United States. More specifically, the current failures in healthcare are failures of primary care - the inadequate system design of the primary care practice, the inadequate reimbursement of primary care, and the poor organization of other health care resources, such as hospitals and specialists around primary care. Note that primary care is not just the primary care provider or even the primary care practice itself. Primary care is an intensive relationship between the patient and her/his primary care practice. The patient is the critical part of primary care.

Although we tend to focus on the problems we face, there are reasons for a great deal of optimism - optimism due to the opportunities we have to improve and redesign care. Medical practice redesign is happening today. It is taking hold and has become a movement that is gaining momentum.

For the first time in history, we have both the knowledge and the capabilities to force substantial change. We are at a unique time in the history. In five or ten years, we might well look back with amazement at the pace of the changes that are currently taking place. The route is clear: We know what to do. We know how to make the system better. The crucial question is whether U.S. health care will have the courage to take on these difficult solutions. When one compares the U.S. health care system with those of other industrialized countries, one is led to the more specific conclusion that the two major problems in U.S. health care are the way we deliver primary care and the way primary care is financed.

A Premise - Our premise is that primary care is the only natural locus of control of health care quality and costs. It is the only entity that is charged with the longitudinal care of the patient. It is the only entity whose job it is to consider the whole patient-the health of the whole person, including mental and physical. While I would not argue that primary care should be all things to all people, it should be designed to achieve much higher performance than it achieves currently. Such a redesign of primary care is possible today.

However, if primary care is not successful in its core tasks of prevention, wellness, and the care of common conditions including many chronic conditions, it will not be possible to control either quality or cost of care in the United States. Again, hospital care and Part-ecialty (specialty) care are crucial to health care, but their use is all too often the failure of upstream care.

Our national focus on disease management programs is a good example of the failure of primary care and the failure of our efforts to improve care. If disease management programs are considered necessary today, it is because primary care is not doing its job. From a primary care perspective, the treatment of chronic conditions, such as diabetes, congestive heart failure, and asthma, is basic and straightforward. The care of these conditions is simply not that difficult. However, the quality failures in the treatment of these conditions are well documented. Disease management programs represent a Band-Aid ${ }^{\oplus}$ approach to problem solving. They acknowledge that there are problems in health care delivery, and instead of addressing those problems directly, these programs create additional, expensive, fragmented responses to the primary problems. For some reason, the healthcare industry has demonstrated an inability to develop a sharp focus on solving core problems. It seems much more willing to create complicated responses to our problems than we are to fix the core problems of our delivery system. Disease management is a perfect example. If primary care is not delivering high quality care for those with chronic conditions, we can either find a way to work around primary care or we can find a way to fix it.

There are far too many efforts focused on working around primary care and not yet enough efforts focused on fundamentally redesigning primary care so that it can deliver the appropriate performance. To spend our limited capital on programs that disable primary care is dangerous and expensive. At the same time, primary care has to take a very different role in terms of accepting accountability and responsibility for its performance. There is no doubt that the current model of primary care is not appropriately designed, and therefore, it is not capable of accomplishing the role that we need it to accomplish; at the same time, the financing system does not support the efforts of primary care. We have continued to devalue the role that the primary care practice performs. This is clearly expressed in terms of reimbursement. The current financing system provides inadequate financing, as well as the wrong incentives, to primary care providers.

But there is a solution, and there are some exciting opportunities. What would it take for primary care to assume that role of the key coordinators and caregivers? It would take a redesign of the primary care system; it would take redesign of the finance system. But with that 
said in the Patient Centered Primary Care Collaborative we have the employers of over 50 million lives, and all the primary care players with a membership over 333,000 physicians from the AAFP ACP AAP and the $\mathrm{AOA}$ at the table and we are designing our primary care system around three key elements.

The first is doctor patient "Relationship." We want to make sure that we provide our patients with fantastic relationship. Great relationship in health care does not just happen spontaneously; it is a product of system design. Our systems must foster the creation and maintenance of effective relationship. To a large degree, care is relationship; it is not only the things that we do, but it is the quality of our interactions. When relationship is disrupted, care suffers.

The second component is patient Centered, the patient's needs at the centre or "Service" If you want to cut out the jargon." The service we buy for our employees should be the best service that patients experience. We know that there is a substantial gap between the service our employees should get and the service that they do get. Examples of service defects include relatively poor access to physicians and the inconvenience of waiting - waiting for appointments, waiting in the medical practice, and waiting for test results, for example.

The third component is "Reliability." How do we create medical practice systems that exude clinical reliability? Reliability, like its cousin, Patient safety is a characteristic of the underlying system design. These components of Patient Centered Primary Care (PCPC) Relationship, Service, and Reliability - are critical to the care process, particularly within primary care. Yet our current medical practices have substantial deficits in each of these areas as has been well described by the Institute of Medicine and others. Getting these components right will be critical to the redesign of primary care if we desire that entity to produce the outcomes of higher quality, more cost effective care. In fact, for the first time in history, we have both the knowledge and the technology to make the achievement of these aims possible. Technology is obviously an important enabler of the characteristics of Relationship, Service, and Reliability.

Example: A critical component of relationship is our ability both to know the patient and to communicate with the patient. Patients feel cared for; they feel the right relationship when people within their medical practice know them. That means that health care workers have to have instantaneous access to patient specific information such as medications, problem lists, social history such as the individual's occupation or avocations, and more. Knowing a patient is the opposite of a patient feeling anonymous.

\section{Electronic systems are necessary for this}

For example, when doctors are taking calls over the weekend and a patient calls in with a problem, they have instantaneous access to that individual's records. The benefits of having such patient specific information readily at hand are obviously in terms of improving safety and quality of care. However, what the patient experiences is a much stronger bond to the practice because they feel known. Therefore, when we are designing medical practices, it is an imperative to consider how our information systems foster relationship by making the most applicable patient-specific information readily available at all times.

\section{Communication is another aspect of relationship}

In today's primary care system, the only way most individuals can communicate with their physician is by scheduling an appointment. It is terribly difficult for patients to get a physician to the telephone to discuss their health concerns or even to answer questions. The current system relies nearly exclusively on the very constrained interactions within a medical visit as the means of communicating with each other. We know how this feels both from a patient and a provider perspective. If we are to improve our communications, we must, therefore, move beyond the visit-based method of care to include other opportunities to interact. The use of much more telephone care, as well as electronic care via email becomes an imperative in a new system design. Patients need the ability to ask their doctor questions in a convenient manner at their own discretion. They need to be able to access their doctor for coaching and counsel when necessary. And we need their doctor to provide them with assistance and appropriate follow-up among other things. Much of this work can occur outside the visit, but it requires open communication systems between patients and their practice. In fact, in Denmark we have found that the proactive use of telephone and electronic care has allowed the Danes to reduce follow-up visits with patients by $50-70 \%$ while maintaining the quality of care and improving our relationships with patients at the same time. Note the significance of that figure - a $50-70 \%$ education in follow-up visits simply by moving to the use of non-visit care mechanisms. A last example pertains to clinical reliability.

Our primary care physicians have to be capable of tracking our patients so that individuals stop falling through the cracks. Diabetics frequently go without the appropriate follow-up or effective preventive measures, because existing practices have no way of specifically tracking these individuals and noting when they are delinquent for care. This ability to proactively manage our patients is critical - the function is called a registry, and they are possible today. Registries, generally electronic, provide us with the ability to place patients within specific groupings for tracking purposes. The appropriate care parameters for those different groups can be defined, and the care of individuals within those groups can be continuously monitored against those standards. Therefore, if a diabetic is due for an important test like and $\mathrm{AlC}$, the practice knows it. If an at-risk patient's numbers are not at a target level, the PCPC practice is aware and can pull the patient into the care process. Such proactive care can only be accomplished by reconstructing the practice around such registries and redesigning the work processes with these capabilities in mind. Such functions are necessary to deliver on the promise of highly reliable care. Contrast such a system with the near purely reactive system that we currently have.

\section{Patient centered primary care collaborative}

We have at the table all 333,000 primary care doctors in the USA right now - IBM was the first employer to engage directly with the primary care providers and we have achieved an agreement from all the Primary care organizations this in the past year (see attached agreement). It is now possible to design medical practice systems that incorporate these characteristics. The system design required is merely hinted at by the provided examples. To design systems around these components fundamentally changes the work that we do in a practice, but those changes are necessary if we are to achieve our desired aims. It is possible to design such systems today, and in this redesign there exists the potential to transform health care in the manner that we all know is necessary. Unfortunately, such effective and efficient care is simply not reimbursed in today's financing environment. Time and time again, we see the lack of financial incentives to do the right thing for the patient within primary care. A primary care practice works hard to improve its service and its clinical reliability, but it receives the exact same reimbursement as all other practices within its area. There 
is no differential payment either for quality of care or for superior service. These problems thwart appropriate system design.

\section{Conclusion}

Primary care is the crux of our health care systems, failings today. If we are to redesign our health care system around primary care, both the financing system and the primary care system have to be redesigned. Such a redesign is possible and could be done so that primary care provides a much higher level of both quality and cost efficiency. Primary care is the only natural place for the overall control of these items, and to design solutions that do not take this entity into primary consideration will ultimately fail to provide us with the performance we need so desperately from our health care system. Financially and quality in care will be more considered.

Note - this has been coordinated with the leadership of the primary physician organizations in the USA the AAFP, ACP, AOA and AAP. The words have been stated by many who understand this issues it is the position of IBM and the position of the Patient Centered Primary Care Collaborative. 